\title{
Effect of the Electrolyte Concentration and Substrate on Conducting Polymer Actuators
}

\author{
Jose G. Martinez, Toribio F. Otero and Edwin Jager
}

\section{Linköping University Post Print}

\section{Tweet}

N.B.: When citing this work, cite the original article.

Original Publication:

Jose G. Martinez, Toribio F. Otero and Edwin Jager, Effect of the Electrolyte Concentration and Substrate on Conducting Polymer Actuators, 2014, Langmuir, (30), 13, 3894-3904.

http://dx.doi.org/10.1021/la404353z

Copyright: American Chemical Society http://pubs.acs.org/

Postprint available at: Linköping University Electronic Press

http://urn.kb.se/resolve?urn=urn:nbn:se:liu:diva-106853 


\title{
Effect of the electrolyte concentration and substrate on conducting polymer actuators.
}

\author{
Jose G. Martinez ${ }^{\dagger, \ddagger}$, Toribio F. Otero ${ }^{\dagger}$, Edwin W. H. Jager ${ }^{{ }^{*}}$ \\ ${ }^{\dagger}$ Universidad Politécnica de Cartagena. ETSII. Center for Electrochemistry and Intelligent Materials (CEMI).Paseo Alfonso \\ XIII, Aulario II, 30203 Cartagena. Spain. \\ ${ }^{\ddagger}$ Linköping University, Department of Physics, Chemistry and Biology, Biosensors and Bioelectronics Centre, 58183 Lin- \\ köping, Sweden.
}

KEYWORDS: Conducting polymers, Artificial muscles, Actuators, Substrate, Electrolyte concentration, Water exchange, Polypyrrole, Electroactive polymers

\begin{abstract}
The effect of the electrolyte concentration ( $\mathrm{NaCl}$ aqueous electrolyte) on the dimensional variations of films of polypyrrole doped with dodecylbenzenesulphonate PPy(DBS) on Pt and Au wires was studied. Any parallel reaction that occurs during the redox polymeric reaction that drives the mechanical actuation, as detected from the coulovoltammetric responses, was avoided by using Pt wires as substrate and controlling the potential limits, thus significantly increasing the actuator lifetime. The $\mathrm{NaCl}$ concentration of the electrolyte, when studied by cyclic voltammetry or chronoamperometry, has a strong effect on the performance as well. A maximum expansion was achieved in $0.3 \mathrm{M}$ aqueous solution. The consumed oxidation and reduction charges control the fully reversible dimensional variations: PPy(DBS) films are faradaic polymeric motors. Parallel to the faradaic exchange of the cations, osmotic, electrophoretic, and structural changes play an important role for the water exchange and volume change of PPy(DBS).
\end{abstract}

\section{INTRODUCTION}

Conducting polymers (CPs) offer an attractive approach to chemical driven mechanical actuators. Actuators built employing CPs work in a similar way as natural muscles and are therefore often addressed as artificial muscles. When an electric pulse arrives to the $\mathrm{CP}$, a chemical reaction occurs varying the composition and properties of the material, including the volume that can be utilized as an actuator. ${ }^{1,2} \mathrm{CP}$ actuators have been demonstrated comprising many different conducting polymers (polyaniline, polypyrrole, poly $(3,4-$ ethylenedioxythiophene)) and dopants. ${ }^{3-6}$ They have been fabricated in a variety of sizes, ${ }^{7}$ from nano- and microscale ${ }^{8,9}$ to centimeters scale ${ }^{1,2}$ and in different configurations offering different kind of movements such as fibers or films producing linear movements, ${ }^{10-12}$ bilayers and trilayers producing angular movements, ${ }^{1,2,13}$ and as bulk material producing perpendicular expansion. ${ }^{14-16}$

Polypyrrole (PPy) doped with dodecylbenzenesulfonate (DBS) is one of the most studied materials for actuators due to its good mechanical properties and the great volume changes occurring when the electrochemical reaction (1) occurs: ${ }^{8,17-19}$

$$
\begin{aligned}
& {\left[\left(P P y^{0}\right)\left(D B S^{-}\right)_{n}\left(C^{+}\right)_{n}(\text { Solv })_{m}\right]_{g e l} \rightleftarrows} \\
& {\left[\left(\text { PPy }^{n+}\right)\left(D B S^{-}\right)_{n}\right]_{s}+n\left(C^{+}\right)+m(\text { Solv })+n\left(e^{-}\right)_{\text {metal }}}
\end{aligned}
$$

(1)

where subscripts mean s solid and aq, aqueous solution, DBSrepresents the charge balancing macro-anion trapped inside the $\mathrm{CP}$ during polymerization, PPy represent the polypyrrole chains and $\mathrm{C}+$ represent a cation. In the reduced state a dense gel-like material (indicated by the subscript gel) containing polymeric chains, ions and solvent molecules (S) is formed.

Electrochemical reaction (1) is the responsible for the dimensional variations (swelling/shrinking during reduction/oxidation, respectively) of the PPy material used for the construction of electrochemical actuators. During the oxidation of the PPy chains, electrons are extracted from the chains generating positive charges, cations and solvent are expelled towards the solution in order 
to keep charge and osmotic balance: the material shrinks. During electrochemical reduction all the processes are reversed, cations and solvent molecules penetrate into the material and the film swells. Being the process driven by reaction 1 the reaction rate $(r)$ is: ${ }^{20}$

$$
\begin{aligned}
& r=\frac{i}{F V}=k \prod c_{j}^{\beta, j} \exp \left(\frac{\alpha n F}{R T}\right)=k^{\prime} \prod c_{j}^{\beta, j} \exp \left(\frac{-E_{a}}{R T}\right) \\
& =k^{\prime}\left[\left(P P y^{n+}\right)\left(D B S^{-}\right)_{n}\right]^{a}\left[N a^{+}\right]^{b}[S]^{c} \exp \left(\frac{-E_{a}}{R T}\right)
\end{aligned}
$$

where $\mathrm{i}$ is the electric current passing through the active PPy (reacting inside the electrolyte), $\mathrm{F}$ is the Faraday constant $\left(\mathrm{F}=96485 \mathrm{C} \mathrm{mol}^{-1}\right), \mathrm{V}$ is the volume of the PPy film in the electrolyte, $\mathrm{k}$ is the rate constant or rate coefficient, $\mathrm{c}$ is the concentration of the reactant $\mathrm{j}, \beta_{\mathrm{j}}$ is the reaction order of the reactant $\mathrm{j}, \alpha$ is the symmetry factor, $\mathrm{n}$ is the number of removed charges per chain, $\mathrm{R}$ is the universal gas constant $\left(\mathrm{R}=8.314 \mathrm{~J} \mathrm{~K}^{-1} \mathrm{~mol}^{-1}\right)$, $\mathrm{T}$ is the temperature, $\mathrm{k}^{\prime}$ is the preexponential factor, $\mathrm{E}_{\mathrm{a}}$ is the activation energy, $\left[\left(\mathrm{PPy}^{\mathrm{n}+}\right)(\mathrm{DBS}\right.$ )$\left._{n}\right]$ is the concentration of oxidized sites on the chains in the polymer film, $\left[\mathrm{Na}^{+}\right]$is the concentration of $\mathrm{Na}$ cations in solution, $[\mathrm{S}]$ is the concentration of the solvent being exchanged during reaction (1) and $\mathrm{a}, \mathrm{b}$ and $\mathrm{c}$ are the reaction orders associated to the oxidized polymer, cations and solvent respectively. Thus, any variable acting on the reaction rate (temperature, electrolyte concentration, solvent, electrode surface, concentration of active centers in the film) is expected to influence the swelling/shrinking rates and the actuating properties of the material.

The effect of the electrolyte concentration on the electrochemical response has been previously used to build concentration sensors. ${ }^{21-25}$ Different methodologies allow following the concentration influence on the electrochemical responses of conducting polymers coating metal electrodes. Laslau et al. used modified scanning ion conductance microscopy. ${ }^{26}$ Bay et $a l$. measured the linear expansion of $\mathrm{PPy}(\mathrm{DBS})$ strips as a function of the electrolyte concentration using a forcedisplacement setup. They observed that the expansion decreased with increasing electrolyte concentration, which they explained by means of the osmotic expansion suffered by a polymer membrane in solution. ${ }^{27}$ Studying the radial expansion of PPy(DBS) Carlsson et al. also obtained a decreasing final expansion with increasing electrolyte concentration. ${ }^{28}$ However, the final expansion at concentrations lower than $0.15 \mathrm{M}$ was extremely slow. When looking at the medium time scale the maximum volume change was obtained in 0.3 M solutions. The expansion rate increased with increasing electrolyte concentration, being fastest at $2 \mathrm{M}$ and slowest at $0.05 \mathrm{M}$. These results indicate the dual nature of the volume change: ion insertion driven by the faradaic current which is dominating at the short time scale and solvent swelling driven by osmotic pressure which dominates at the longer time scale, thus resulting in an optimum expansion at $0.3 \mathrm{M}$ for medium time scales.

Jo et al. found a maximum in the deflection of IPMC actuators with the concentration explained because of the osmotic presssure differences due to the different number of ions in the surface. ${ }^{29}$ Skaarup et al. have attributed the different amount of exchanged solvent during actuation to the cation solvation number and to the different osmotic pressure in different electrolyte concentrations. ${ }^{30,31}$

Moreover the electrolyte concentration, also the substrate material and the solvent can play an important role on the actuation. When the voltammetric results in aqueous solutions from CP films coated on metals were represented as coulovoltammetric responses, the irreversible generation of hydrogen at the polymer/metal interface was detected and quantified at low cathodic potentials. ${ }^{32}$

Here, we revisit the effect the electrolyte concentration on the actuation profile of the PPy(DBS) on metal substrates. Our custom-built set-up is based on a Laser Scan Micrometer (LSM) and allows for real time, non-contact measurements of the $\mathrm{PPy}(\mathrm{DBS})$ volume change during the redox reaction in deep detail ${ }^{33}$ thus giving new insights in the mechanisms of the volume change of PPy. The potential range used in this study was selected in order to avoid parallel reactions caused by the substrate material. The final aim is to identify the different reactions and processes playing a key role in actuation in order to get improved electrochemical actuators and to develop new applications and products.

\section{EXPERIMENTAL SECTION}

Sodium chloride ( $\mathrm{NaCl}$, from Merck) and Sodium dodecylbenzenesulfonate (NaDBS, from TCI Europe for electrogeneration and Aldrich for characterization) were used as received. Pyrrole (Sigma-Aldrich) was distilled before used under vacuum and stored at $-18{ }^{\circ} \mathrm{C}$ under nitrogen atmosphere. Ultrapure water was obtained from Millipore Milli-Q equipment.

Gold (Ø $0.5 \mathrm{~mm})$ and platinum $(\varnothing 1 \mathrm{~mm})$ wires from Goodfellow were used as the working electrodes. The wires were electrically insulated with an electrically insulating heat-shrink polymer, but letting a length of $10 \mathrm{~mm}$ in the middle of the wire uncoated. Each of those wires was used for the polypyrrole electrodeposition. The counter electrode was a cylindrical gold coated plastic film, ensuring a uniform electrical field around the working electrode and thus a uniform PPy coating. The gold counter electrode was constructed by first depositing a chromium layer, $3 \mathrm{~nm}$ thick, onto an acetate sheet. On this adhesion layer a gold film, $200 \mathrm{~nm}$ thick, was then deposited. This flexible material was cut into the right shape to fit the electrochemical cell.

For the electrogeneration, a cylindrical electrochemical cell with a diameter of $2 \mathrm{~cm}$ was used. The working electrode was 
set in the center of the cell, surrounded by the counter electrode. A silver/silver chloride $(\mathrm{Ag} / \mathrm{AgCl})$ reference electrode from BASi was used and located near the upper part of the working electrode. Every potential in this work is referenced to this reference electrode. The polypyrrole coating was obtained in $8 \mathrm{~mL}$ of $0.1 \mathrm{M} \mathrm{NaDBS}$ and $0.1 \mathrm{M}$ pyrrole aqueous solution by applying a constant potential of $0.55 \mathrm{~V}$ versus $\mathrm{Ag} / \mathrm{AgCl}$, during the time required to consume a constant charge of $140 \mathrm{mC}$. The procedure was repeated obtaining reproducible films: $6.12 \pm 1.08 \mu \mathrm{m}$ thick and $0.23 \pm 0.06 \mathrm{mg}$ on gold and $3.12 \pm 0.51 \mu \mathrm{m}$ thick and $0.21 \pm 0.03 \mathrm{mg}$ on platinum.

After generation the coated electrode was immersed in water during 20 seconds and then was dried for 3 minutes in air. The thickness of the polymer films were determined by difference between the diameter of the coated and uncoated wire, both measured with a Laser Scan Micrometer (LSM), keeping the position of the electrode constant. Then the polypyrrole coated electrode was weighed and the polypyrrole mass was obtained by mass difference between coated and uncoated electrode using a Sartorious BP210D balance (precision $10^{-5} \mathrm{~g}$ ).

The electrochemical characterization and parallel determination of the induced dimensional variation, i.e. the expansion of the PPy layer, was performed in a transparent plastic cell of 50 $\mathrm{mL}$ with a rectangular cross-section. A flat and rectangular platinized titanium electrode was used as counter electrode. The electrolyte was a $0.1 \mathrm{M} \mathrm{NaCl}$ aqueous solution, which was filtrated through a $0.2 \mu \mathrm{m}$ filter to remove any potential particulate matter than could interfere with the LSM.

Electrochemical experiments were performed with a potentiostat-galvanostat Autolab PGSTAT-20 attached to a personal computer with GPES software.

Diameter variations were measured with a LSM from Mitutoyo (Mitutoyo LSM-501H) controlled by means of a display unit (Mitutoyo LSM-6100). From these diameter variations the diametrical (or out-of-plane or perpendicular) expansion and strain of PPy could be calculated as described in Fig 1. In order to obtain dynamic measurements, the output signal of the LSM (diameter of the working electrode) was fed to the potentiostat where it was recorded simultaneously with the electrochemical experiments. More details about the LSM setup can be found in reference ${ }^{33}$.

\section{RESULTS}

\section{Influence of the Substrate}

\subsection{Uncoated metals}

Gold and Pt wires and the wires coated with PPy(DBS) were checked by cyclic voltammetry in $0.0375 \mathrm{M} \mathrm{NaCl}$ aqueous solution. Voltammetric responses obtained between -1.25 and
$0.5 \mathrm{~V}$ at $20 \mathrm{mV} \mathrm{s}^{-1}$ (Fig. 2a) from the uncoated metal wires show the flow of higher currents through the gold wire than through the platinum wire. The coulovoltammetric responses (Fig. 2b) from the uncoated metal wires were obtained by integration of the voltammograms from figure $2 \mathrm{a}$.

Coulovoltammograms are representations of the accumulated consumed charge during cyclic voltammetry starting from zero charge at the initial time allowing the detection and of parallel irreversible reactions, such as water electrolysis at the metal/polymer interface. ${ }^{32}$ Oxidation reactions promote positive increments in the consumed charge while reduction reactions promote negative increments in the consumed charge. Reversible redox processes give a closed loop in the coulovoltammetric response. Irreversible processes give open parts corresponding to the charge (final minus initial charge) consumed by an irreversible process. The coulovoltammetric responses (Fig. 2b) corroborate the presence of irreversible reduction reaction processes (a negative irreversible consumed charge) in both electrodes: reduction charges are greater than oxidation charges. Irreversible reduction charges, and the concomitant irreversible reaction (e.g. hydrogen evolution from aqueous solutions: it disappears from dry organic solvents ${ }^{32,34}$ ), are higher from the gold electrode than from the Pt electrode in the studied potential rage. The irreversible reduction reaction starts at $-0.8 \mathrm{~V}$ on Pt. On gold an initial irreversible shoulder is initiated at $0.0 \mathrm{~V}$ on the cathodic branch of the voltammogram with a second irreversible reaction at more cathodic potentials than $-0.8 \mathrm{~V}$. Those irreversible reactions can be the origin of the parallel degradation of both the polymer film electroactivity and the actuation of the artificial muscles.

\subsection{Coated metals electrochemistry}

In order to try to avoid these irreversible reactions the responses from PPy(DBS) coated Pt and Au electrodes were investigated by potential cycling between the cathodic limits of $-1 \mathrm{~V}$ or $-0.8 \mathrm{~V}$, respectively, and the anodic limit of $0 \mathrm{~V}$ at 20 $\mathrm{mV} \mathrm{s}^{-1}$. The voltammetric and coulovoltammetric responses for $1^{\text {st }}$ and $150^{\text {th }}$ cycle are shown in figures $3 \mathrm{a}$ and $3 \mathrm{~b}$ for the $\mathrm{PPy}(\mathrm{DBS})$ on Au electrode and figures $3 \mathrm{c}$ and $3 \mathrm{~d}$ for PPy(DBS) on Pt. An irreversible charge (open coulovoltammetric part) is present in the response of PPy(DBS) on $\mathrm{Au}$ for the studied potential limits, see fig. $3 \mathrm{~b}$ as an example. The irreversible charge in the response of PPy(DBS) on Pt only was present for more cathodic potential limits than $-0.8 \mathrm{~V} .{ }^{32}$ The closed coulovoltammetric loop obtained for this potential limit (fig. 3d) indicate that, by cycling the coated electrode between -0.8 and $0.0 \mathrm{~V}$ in aqueous solution, the film oxidation charge equals the film reduction charge and no parallel irreversible reactions occur in the studied potential range. Therefore $-0.8 \mathrm{~V}$ was selected as the cathodic potential limit for the subsequent experiments being sure now that the full charge involved in experiments is only devoted to oxidize and reduce 
the PPy(DBS) film inducing the subsequent dimensional variations.

\subsubsection{Dimensional variations during cycling}

Under the above mentioned experimental conditions the variation of the electrode diameter was followed during consecutive potential cycles resulting in a reversible oxidation/reduction of the PPy(DBS) films. Figures $3 e$ and $3 f$ show the diametrical strain (i.e. relative thickness change) of PPy along the stationary potential cycle. As expected from any conducting polymer exchanging cations for charge balance (reaction 1) the PPy thickness decreases during oxidation (reaction 1 forwards, with flow of positive current on the voltammograms or positive increment of the charge along the coulovoltammograms) and increases during reduction (reaction 1 backwards, with flow of negative voltammetric current, or negative increment of the charge along the coulovoltammogram). The large reversible expansion of $\sim 30 \%$ is similar to previous reports. . $^{14,28,33}$ The remarkable result now is that after 150 cycles almost the same dimensional variations are observed while in previous descriptions of large linear dimensional variations the range of the dimensional change used to decreased down until around $1 \%$ after a few cycles. ${ }^{11,35,36}$

\subsubsection{Actuator degradation by parallel irreversible} reactions

In order to check the influence of the parallel reactions on the lifetime of the polypyrrole actuator, both coated electrodes ( $\mathrm{Au}$ and $\mathrm{Pt}$ ) were submitted to 150 consecutive square potential waves (reduction potentials of $-1 \mathrm{~V}$, or $-0.8 \mathrm{~V}$, for $200 \mathrm{~s}$; followed by the anodic potential step to $0 \mathrm{~V}$ kept for $200 \mathrm{~s}$ ). The electrode was checked, by cyclic voltammetry at $20 \mathrm{mV} \mathrm{s}$ ${ }^{1}$ before and after each treatment. Voltammetric (Fig. 3a from $\mathrm{Au}$, Fig. 3c from Pt) and chronocoulommetric (Fig. 3b, Au; Fig. 3d, Pt) responses as well as the simultaneous diametrical (or perpendicular/out-of-plane) expansion of the PPy layer coated on the electrode (Figure $3 \mathrm{e}$ from $\mathrm{Au}$; $3 \mathrm{f}$ from $\mathrm{Pt}$ ) measurements were recorded. After the square potential waves from $-1 \mathrm{~V}$ to $0 \mathrm{~V}$, a degradation of the PPy(DBS) layer on the cylindrical Au electrode (Fig. 3b) was observed: the voltammetric charge after 150 cycles decreased to $60 \%$ compared to that of the control before cycling. A similar drastic decrease of the PPy(DBS) diametrical strain was observed (Fig 3e). However, using a Pt substrate and a cathodic potential limit of $0.8 \mathrm{~V}$, the voltammetric, coulovoltammetric and PPy(DBS) strain curves before and after 150 cycles overlapped (Fig $3 \mathrm{~d}$, f), indicating fully reversible reactions.

In conclusion, by limiting the cathodic potential to $-0.8 \mathrm{~V}$ and using a Pt electrode irreversible reactions at the metal/polymer interface are avoided and the actuation of PPy(DBS) remains stable under potential cycling: the actuation lifetime is significantly increased.

\section{Influence of the electrolyte concentration}

\subsection{Voltammetric responses}

The expansion of PPy(DBS) on the Pt wire was studied by consecutive potential sweeps in different concentrations of $\mathrm{NaCl}$ aqueous solutions at $20 \mathrm{mV} \mathrm{s}^{-1}$. The steady state voltammetric responses attained after three consecutive potential cycles are shown in Fig. 4a. Similar anodic responses were obtained for the different concentrations, with the oxidation maximum around $-0.35 \mathrm{~V}$. The reduction maximum shows a strong influence of the electrolyte concentration, shifting anodically when the electrolyte concentration increases up to $2 \mathrm{M}$ and then cathodically until $3 \mathrm{M}$. As a consequence, the potential difference between the anodic and the cathodic maxima decreases (Table 1) when the concentration increases until $2 \mathrm{M}$ and then increases for $3 \mathrm{M}$. Those gradients indicate the ionic resistance gradient between the cation's expulsion (anodic, oxidation) and its entrance (cathodic, reduction). During the anodic potential sweep the material starts from a similar swollen and reduced state every time and the influence of the electrolyte concentration on the material oxidation and expulsion of the cations (reaction 1 forwards) towards the solution is very minor. During the cathodic potential sweep the material reduction (reaction 1 backwards) begins from a shrunk and conformational packed structure. The experimental results indicate that the electrolyte concentration has only minor influence on producing shrunk structures, not influencing the entrance of cations during reduction. The reduction maxima shifts to lower overpotentials when the cation's concentration increases, as expected for any electrochemical reduction: since the cation from the electrolyte is a reactant of the reaction 1 , the increase of a reactant concentration is expected to give rising reaction rates (Eq. 2) at lower overpotentials. As a further matter, rising electrolyte concentrations are expected to give rising ionic conductivities of the solution and rising ionic diffusion rates due to the rising gradients. ${ }^{32}$

\subsection{Coulovoltammetric responses}

As expected from the selected potential range, closed stationary coulovoltammetric responses were obtained after three consecutive potential cycles for each electrolyte concentration used (Fig. 4b): only reversible redox processes in the PPy film were involved. The film redox charge is the difference between the coulovoltammetric loop minimum and its maximum. In the studied potential range, the redox charge first increases with the electrolyte concentration up to 0.15-0.3 M and then decreases for higher concentrations (Fig. 4b and Table 2). Different redox charges indicate that only a fraction of the film polymer chains participate in the oxidation/reduction reactions and that the electrolyte concentration promotes a shift of the fraction magnitude. That means that a fraction of the film, even if the chemical (electrolyte presence) and electrical (applied potential range) conditions are suitable 
for the reaction, does not react due to some structural restrictions during the reactions. The reaction driven conformational movements of the chains generate the required free volume to lodge balancing cations and water. Those conformational movements also are expected to be influenced by the solvent, acting as a plasticizer. In this way shifts of the oxidation charge from Table 2 could point to some similar shifts on the relative water content in the most reduced state of the material attained at the cathodic potential limit.

\subsection{The full dimensional changes are faradaic processes}

The expansion of the PPy(DBS) layer on the Pt wire follows loop evolutions parallel to those observed for the coulovoltammetric responses (Fig. 4c).The total expansion (difference between the loop maximum and the loop minimum) induced by the electrochemical processes in the film also presents a maximum at $0.3 \mathrm{M}$.

By combining the consumed charge (Fig. 4b) and the PPy expansion (Fig. 4c) data, the evolutions of the PPy dimensional changes as a function of the consumed charge (Fig. 4d) were obtained. During the film reduction (swelling) the diameter increases linearly with the charge, see Fig. 4d. These results corroborate the faradic nature of the actuation;

$$
\Delta d=k \Delta Q
$$

where $\Delta \mathrm{d}$ is the thickness change (expansion) and $\Delta \mathrm{Q}$ the consumed charge and $\mathrm{k}$ is a constant characteristic of the system (polymer, solvent and salt), as previously was obtained from bending CP actuators. ${ }^{37}$ Reaction 1 drives the involved charge $(\mathrm{Q})$ and the dimensional variations (free volume generation) required to lodge/expel cations (one $\mathrm{Na}^{+}$per charge) and water molecules.

\subsection{Dimensional variations at the intermediate states in-} forms about the relative water exchange

Fig. 4d represents the transition between two quasi-stationary oxidation states indicating that the full process is a faradaic process. The linear variation of the actuator dimensions during the cathodic potential sweep indicates the parallel entrance of cations and water, with the same number of water molecules per unit of charge, during the polymer reduction.

During oxidation (shrinking), this variation is not linear. A faster thickness change occurs at the beginning of the oxidation, the shrinking rate per unit of charge (slope at any point) is always greater than the constant slope attained during reduction. The extra volume change per unit of charge must be due to water (not cation) draining. Thus, a fast solvent draining, probably by electrophoresis combined with some structural change in the film (in a similar way to the drying processes during the industrial electrodeposition of paint films) ${ }^{38}$ is proposed as origin of the observed fast dimensional changes. The thickness variation at the end of the potential cycle is much slower pointing to that, as most of the water was drained, here the major part of the shrinking is caused by expulsion of cations with a smaller amount of water per charge unit. The closed loop indicates that the overall number of expelled cations and water molecules during oxidation equals those entering during reduction. A better experimental definition of the slopes could allow the quantification of the instantaneous solvent exchanged per unit of charge. The fast evolution of the dimensions at the beginning of the anodic potential sweep indicates that most of this solvent is required for osmotic balance with a minor fraction linked to the cation (solvated water).

Table 2 summarizes the dimensional variations and the involved charges from Fig. 4. As can be seen the expansion of PPy increases from $0.0375 \mathrm{M}$, reaches a maximum at $0.3 \mathrm{M}$ and thereafter decreases. The same evolution can be seen for the consumed charge having a maximum at 0.15-0.3 M. Surprisingly, the film expansion per unit of charge also presents a maximum at $0.3 \mathrm{M}$. Following the previous paragraph this results should indicate that the number of water molecules exchanged per unit of charge (per exchanged $\mathrm{Na}^{+}$, or per generated or eliminated active center on the polymer chain) involved in the reaction also changes with the electrolyte concentration in parallel to the total charge evolution.

It can be summarized that the dimensional variation of the PPy film during reduction in $\mathrm{NaCl}$ aqueous solutions is due to the parallel insertion of cations and solvent. The relative insertion of both components changes with the electrolyte concentration getting the maximum expansion efficiency per unit of charge, thus the higher amount of exchanged solvent per unit of charge, at $0.3 \mathrm{M}$. During the polymer oxidation electrophoretic and structural processes, produce a fast solvent draining per unit of charge at the beginning of the oxidation and a very low solvent draining per unit of charge at the end of the oxidation.

\section{Chronoamperometric results}

The PPy(DBS) redox reactions and dimensional changes were now checked by submitting the electrode to 8 consecutive potential steps from a constant reduction potential $(-0.8 \mathrm{~V})$, kept for $150 \mathrm{~s}$, to a constant oxidation potential of $0 \mathrm{~V}$, kept for $150 \mathrm{~s}$, in each of the studied electrolyte concentrations. In this way any structural memory is erased during the initial square waves resulting in reproducible chronoamperometric responses from the final waves. Before and after this treatment in every concentration the state of the film was checked from the reproducible voltammetric responses in $0.1 \mathrm{M} \mathrm{NaCl}$. When the charge of the voltammetric post-control differs more than $5 \%$ from that of the voltammetric pre-control, a new film was used for the experiments. 
The steady state chronoamperometric responses from the different studied concentrations are shown in Fig. 5a (film oxidation) and Fig. 5b (film reduction). Both anodic and cathodic responses show a maximum and shoulders (see inset figures), which are far away from the expected Cotrell responses for diffusion-controlled processes. Chronoamperometric shoulders and maximums are characteristics responses when structural molecular (conformations) or macroscopic (swelling/shrinking, compaction/relaxation) transformations during both oxidation and reduction reactions. ${ }^{32}$ The conformational relaxation model predicts the polymeric shrinking and subsequent conformational compaction by cation expulsion during the anodic oxidation. This fact should explain the presence of a nucleation/relaxation shoulder or maximum on the cathodic chronoamperometric branches, indicating the entrance of cations through those points (nuclei) of the polymer/solution interface where the chains have a greater mobility. ${ }^{39}$ The presence of similar maxima or shoulders on the anodic responses indicates that the swollen reduced polymer PPy $\left(\mathrm{DBS}^{-}\right)_{\mathrm{n}}\left(\mathrm{Na}^{+}\right)_{\mathrm{n}}(\mathrm{S})_{\mathrm{m}}$ presents an energetically stable structure, still not described by the literature. The oxidation and transformation of the reduced structure begins by oxidation-nucleation, as deduced from the characteristic nucleation maximum on the anodic chronoamperogram. The electrolyte concentration influences the kinetics of both, oxidation and reduction, structural changes as deduced (Inset Figs. $5 \mathrm{a}$ and $5 \mathrm{~b}$, respectively) from the initial current steps.

The parallel PPy dimensional changes as function of time for the different electrolyte concentrations is shown in Fig. 5c (film oxidation) and Fig. 5d (film reduction). A faster expansion is observed at the beginning of the actuation, corresponding with the initial current peak. After few seconds, a slow expansion is observed. Slopes from Figs. $5 \mathrm{c}$ and $5 \mathrm{~d}$, obtained from the average values of several points to minimize noise, give the expansion rate shown in Figs. 5e and 5f. The inset figures present the evolution of those expansion rates during the initial 10 seconds of oxidation and reduction, respectively. By comparing the insets from Figs. 5a vs. 5e and from Figs. $5 \mathrm{~b}$ vs. $5 \mathrm{f}$ a good correlation can be noticed between the flowing current (charge per unit of time) and the expansion rate, as expected for a faradaic process.

Following the procedure above described for the voltammetric responses, the consumed oxidation and reduction charges were obtained by integration of the chronoamperometric responses and compared with the concomitant expansions and initial expansion rates in every electrolyte concentration (Fig. 5g). As before, a good correlation was attained and we see the same maximum at $0.3 \mathrm{M}$ occurring for charge, expansion and expansion rate.

\section{DISCUSSION}

The attained experimental results have proved the important influence of the irreversible processes, occurring at the conducting polymer-metal interface, on the film responses and on its lifetime. A significant degradation (60\% of the initial film redox charge) is observed when the PPy(DBS) material was deposited on a gold wire and cycled ( 150 cycles) in the potential range from $-1 \mathrm{~V}$ to $0 \mathrm{~V}$. The observed effect of the metal substrate and the potential range on the occurrence of irreversible reaction promotes a fast degradation of the actuating material. Those results suggest that the electrochemical studies of conducting polymers coated on metals (as electrodes or as part of devices: for Electrochemical Quartz Crystal Microbalance (EQCM) experiments, membranes, actuators, batteries, and so on) in aqueous solutions from the literature, and their conclusions, should require some reconsideration looking for the possible presence of the irreversible hydrogen release.

Both, cyclic voltammetric results (Fig. 4d) and chronoamperometric results (Fig. $5 \mathrm{~g}$ ) show a linear relationship between the consumed charge $(\Delta \mathrm{Q})$ and the dimensional variations (expansion/contraction, $\Delta \mathrm{d}$ ) of the PPy(DBS) film (Eq. 3) for all the studied concentrations. This is the expected result if the dimensional variation is induced by reaction 1 , corroborating the faradaic origin of the dimensional changes in linear CP actuators. Similar faradaic results have been found from bending polymeric actuators constituted by different CPs and in different electrolytes. ${ }^{37}$

A maximum dimensional variation per unit of charge (Table

2 ) is attained when the concentration ranges between 0.15 and $0.3 \mathrm{M}$. The same evolution can be seen for the consumed charge having a maximum at $0.15-0.3 \mathrm{M}$, which would be expected since PPy behaves as a faradaic motor. Interestingly, also the expansion per unit of charge presents a maximum at $0.3 \mathrm{M}$. This clearly shows that the reversible dimensional variation of PPy films driven by electrochemical reactions has two components: insertion of cations and insertion of solvent. Subsequently at $0.3 \mathrm{M}$ both more charge (cations) and more solvent per unit of charge is exchanged between the film and the solution.

This result indicates that a different amount of water molecules are exchanged per unit of charge from electrolytes having different salt concentrations. The decrease in expansion per charge from $0.3 \mathrm{M}$ to $3 \mathrm{M}$ follows what would be expected for an osmotic exchange: the concentration difference of the cations between the solution and the polymer decreases, decreasing the osmotic pressure and less solvent penetrates from the solution per unit of oxidation charge. ${ }^{30,31}$ The subsequent lower swelling ration gives a more stiff polymeric structure (the solvent acts as a plasticizer), less polymer chains are available for oxidation/reduction inside the film and the charge involved to oxidize and reduce the film inside the same potential range decreases. 
Reaction 1 and the subsequent reduction rate (Eq. 2) can explain the attained results: When the electrolyte concentration increases the film reduction rate ( $r$ ) increases, the equilibrium becomes more and more shifted towards the reduced state, $(\mathrm{PPy})\left(\mathrm{DBS}^{-}\right)_{\mathrm{n}}\left(\mathrm{Na}^{+}\right)_{\mathrm{n}}\left(\mathrm{H}_{2} \mathrm{O}\right)_{\mathrm{m}}$ driving more ions and water $(\mathrm{m}$ subindex) per unit of time into the film to give a softer and wetter film. The easier conformational movements due to the presence of a higher content of water inside the film allow a deeper film oxidation and reduction: the average oxidation charge per chain (n subindex) increases with the electrolyte concentration.

By increasing the electrolyte concentration the percentage of free water molecules, $\left[\mathrm{H}_{2} \mathrm{O}\right]$, in solution decreases: it takes part of the solvated ions. Apparently when the salt concentration overcomes $0.3 \mathrm{M}$ the concentration of free water, $\left[\mathrm{H}_{2} \mathrm{O}\right]$,becomes so low that it starts to influence the reaction rate (Eq. 2). Lower concentration of free water give lower reaction rates less water penetrate into the film (lower osmotic pressure) the plasticity decreases and the reaction deep (the involved charge) decreases.

The electrolyte concentration also has an important influence on the asymmetric potential shift of the maxima on the voltammetric results (Fig. 4a and Table 1) and chronoamperometric responses (Fig. 5a and 5b). In PPy(DBS) the polymer oxidation promotes the faradaic (Fig. 4d) film shrinking, closing and compaction by expulsion of counterions and water. ${ }^{32,40}$ The subsequent film reduction with the entrance of cations starts by nucleation-relaxation giving a maximum or a shoulder on the chronoamperometric responses. Faster nucleationrelaxation processes occur when the concentration rises up to 0.6-1M. Then for higher concentration the chronoamperograms show lower currents indicating that a lower fraction of the polymer film participates in the reaction.

The anodic chronoamperograms, Fig. 5a inset, corroborate that the reduced and swollen material PPy $\left(\mathrm{DBS}^{-}\right)_{\mathrm{n}}\left(\mathrm{Na}^{+}\right)_{\mathrm{n}}(\mathrm{S})_{\mathrm{m}}$ also presents an energetically stable structure that only can be broken to expel the balancing cations towards the solution by a slow nucleation initiated processes. The physical-chemical characterization of this second structure, still not studied, requires a deep analytical and theoretical treatment that is being performed by Otero's group.

Both structural processes (the anodic shrinking and packing and the unknown cathodic stable structure) are interconnected. More packed anodic structures and more stable reduced and swollen structures obtained from the lower studied concentrations obstruct the subsequent polymer oxidation/reduction, giving (Table 2, Figs. 5a, 5b and 5g) slow subsequent oxidation and reduction processes and low film reaction charges. The increase of the electrolyte concentration up to $0.15 \mathrm{M}$ favors the structural transformations giving: rising charges (Figs. 4b and 5g), lower potential gradients between oxidation and reduction voltammetric maxima, greater initial chronoamperometric currents and faster reactions (lower times for the chronoamperometric transitions (insert Figs. 5a and 5b). From there, higher concentrations promote decreasing oxidation and reduction charges here attributed to the lower content of free water in the electrolyte.

The linear relationship observed (Fig. 4d) between the reduction voltammetric charge and the diameter increase (swelling) was not kept during anodic potential sweep. During oxidation (shrinking) a fast decrease of the diameter is observed at the beginning of the anodic potential sweep. The diameter decreases faster than expected from the number of expelled ions, obtained from the charge thorough the Faraday's law: n (mol) $=\mathrm{Q}(\mathrm{C}) / \mathrm{F}\left(\mathrm{C} \mathrm{mol}^{-1}\right)$. This fast decrease of the actuator diameter indicates fast solvent draining by electrophoresis: each expelled cation pushes outside the film the water present in front of it. A similar effect is being used for decades to get dry industrial paint films by electrodeposition. ${ }^{38}$ The diameter variation rate at the end of the potential cycle is much slower indicating the expulsion of a lesser amount of solvent per cation. A better experimental definition of the slopes could allow the quantification of the solvent exchanged per cation along the potential cycle. The uniform variation of the actuator dimensions during the cathodic potential sweep indicates a parallel entrance of cations and water (the same number of water molecules per cation) during the reduction. The fast evolution of the dimensions at the beginning of the anodic potential sweep indicates that most of this solvent is required for osmotic balance and with a minor fraction linked to the cation (solvated water). ${ }^{37}$

Furthermore the electrolyte concentration influences, inside the studied potential ranges, the involved redox charge and, therefore, the faradaic composition, $\mathrm{PPy}\left(\mathrm{DBS}^{-}\right)_{\mathrm{n}}\left(\mathrm{Na}^{+}\right)_{\mathrm{n}}(\mathrm{S})_{\mathrm{m}}$, of the polymer film. It also influences the extension of physical processes as electro-osmotic and electrophoretic rates along the reactions as indicated by voltammetric, coulovoltammetric, chronoamperometric and electrochemomechanical results.

\section{CONCLUSIONS}

The actuation profile of PPy(DBS) coated wires was studied in different $\mathrm{NaCl}$ aqueous electrolyte concentrations and on different substrates: Pt and Au wires.

The substrate influences the presence of irreversible reactions, such as the water electrolysis at low cathodic overpotentials, in parallel to the polymer redox reactions. Faster polymer degradations, with loss of involved charge, were observed induced by those parallel reactions influencing the polymer dimensional variations and the actuator lifetime. This irreversible reduction reaction start at $0.0 \mathrm{~V}$ on $\mathrm{Au}$ and at $-0.8 \mathrm{~V}$ on Pt electrodes coated with PPy(DBS) films. By using a Pt electrode and keeping potential range between $0.0 \mathrm{~V}$ and $-0.8 \mathrm{~V}$, those paral- 
lel reactions are drastically reduced and the lifetime of $\mathrm{PPy}(\mathrm{DBS})$ is significantly increased. These results suggest that the electrochemical studies of conducting polymers coated on metals (as electrodes or as part of devices: for Electrochemical Quartz Crystal Microbalance experiments, membranes, actuators, batteries, and so on) in aqueous solutions from the literature, and their conclusions, should require some reconsideration looking for the possible presence of the irreversible hydrogen release.

The effect of the electrolyte concentration on the reversible dimensional variations (diametrical or radial expansion) of the $\mathrm{PPy}(\mathrm{DBS})$ coated $\mathrm{Pt}$ wires was studied in the potential range of 0 to $-0.8 \mathrm{~V}$ by different electrochemical techniques: cyclic voltammetry and chronoamperometry. The change of the diameter between two redox states is a faradaic process, which means that occurs under control of the exchanged charge (reaction 1).

The expansion of PPy(DBS) shows a maximum at an electrolyte concentration of $0.3 \mathrm{M}$. The same maximum was seen for the consumed charge, the expansion per unit of charge, and expansion rate. From those results it is deduced that the reaction driven (Eq. 2) volume change of the PPy film has two main components: exchange of cations and exchange of solvent. At rising salt concentrations, rising $\left[\mathrm{Na}^{+}\right]$, a higher percentage of water is linked to the ions (solvated water) decreasing the availability of free water, $\left[\mathrm{H}_{2} \mathrm{O}\right]$, in solution. The effect of both concentration variations on the expansion intersects at $0.3 \mathrm{M} \mathrm{NaCl}$ aqueous solution resulting in higher film reaction charge (exchanged cations) and higher amount of solvent exchanged per unit of charge.

Voltammetric and chronoamperometric responses indicate the presence and participation of two different and energetically stable structures of the film: an oxidized structure of packed conformations described by the ESCR model and a new reduced and swollen structure still not described by the literature. They are transformed one into the other by reduction or oxidation, respectively, under initial nucleation-relaxation processes and including structural conformational relaxation, swelling, shrinking and compaction process. Both structures are deeply influenced by the electrolyte concentration. The solvent exchange plays an important role during the reaction induced dimensional variations due to the presence of simultaneous osmotic (reduction) and electrophoretic (oxidation) process. The solvent has a direct effect on the volume change through the osmotic pressure and an indirect effect, as plasticizer, influencing the electrochemically induced conformational movements, the charge transfer rate and the fraction of the film chains participating on the reaction.

These studies elaborate the complex process of the volume change of PPy where several processes (charge transfer, ion influx, solvent influx, conformational changes) all occur not only in parallel but also influence each other.

\section{AUTHOR INFORMATION}

\section{Corresponding Author}

* Edwin W. H. Jager. E-mail: edwin.jager@liu.se

\section{ACKNOWLEDGMENT}

Authors acknowledge financial support from the Spanish Government (MCINN) Project MAT2011-24973 and the European Science Foundation COST Action MP1003 European Scientific Network for Artificial Muscles (ESNAM), COST-STSM-

MP1003-11575 and -11581, and Linköping University. J.G. Martinez acknowledges the Spanish Education Ministry for a FPU grant (AP2010-3460). E.W.H. Jager wishes to express his gratitude to Prof Anthony Turner (LiU, IFM) for his support.

\section{REFERENCES}

(1) Otero, T. F.; Angulo, E.; Rodríguez, J.; Santamaría, C. Electrochemomechanical Properties from a Bilayer: Polypyrrole / Non-Conducting and Flexible Material - Artificial Muscle. J. Electroanal. Chem. 1992, 341, 369-375. Pei, Q.; Inganas, O. Conjugated Polymers and the Bending Cantilever Method - Electrical Muscles and Smart Devices. Adv. Mater. 1992, 4, 277-278.

(3) Li, D.; Huang, J.; Kaner, R. B. Polyaniline Nanofibers: A Unique Polymer Nanostructure for Versatile Applications. Acc. Chem. Res. 2009, 42, 135-145.

(4) Khaldi, A.; Plesse, C.; Soyer, C.; Cattan, E.; Vidal, F.; Legrand, C.; Teyssie, D. Conducting Interpenetrating Polymer Network Sized to Fabricate Microactuators. Appl. Phys. Lett. 2011, 98, 164101.

(5) Kaneto, K.; Kaneko, M.; Min, Y.; Macdiarmid, A. Artificial Muscle - Electromechanical Actuators Using Polyaniline Films. Synth. Met. 1995, 71, 2211-2212.

(6) Gandhi, M.; Murray, P.; Spinks, G.; Wallace, G. Mechanism of Electromechanical Actuation in Polypyrrole. Synth. Met. 1995, 73, 247-256.

(7) Long, Y.-Z.; Li, M.-M.; Gu, C.; Wan, M.; Duvail, J.-L.; Liu, Z.; Fan, Z. Recent Advances in Synthesis, Physical Properties and Applications of Conducting Polymer Nanotubes and Nanofibers. Prog. Polym. Sci. 2011, 36, 1415-1442. Micrometer-Size Structures. Science 1995, 268, 1735-1738. Jager, E. W. H.; Inganas, O.; Lundstrom, I. Microrobots for Micrometer-Size Objects in Aqueous Media: Potential Tools for Single-Cell Manipulation. Science 2000, 288, 23352338.

Spinks, G. M.; Liu, L.; Wallace, G. G.; Zhou, D. Z. Strain Response from Polypyrrole Actuators under Load. Adv. Funct. Mater. 2002, 12, 437-440.

Hara, S.; Zama, T.; Takashima, W.; Kaneto, K. Artificial Muscles Based on Polypyrrole Actuators with Large Strain and Stress Induced Electrically. Polym. J. 2004, 36, 151161. 
(12) Yamato, K.; Kaneto, K. Tubular Linear Actuators Using Conducting Polymer, Polypyrrole. Anal. Chim. Acta 2006, 568, 133-137.

(13) Vidal, F.; Popp, J. F.; Plesse, C.; Chevrot, C.; Teyssie, D. Feasibility of Conducting Semi-Interpenetrating Networks Based on a Poly(ethylene Oxide) Network and poly(3,4Ethylenedioxythiophene) in Actuator Design. J. Appl. Polym. Sci. 2003, 90, 3569-3577.

(14) Smela, E.; Gadegaard, N. Surprising Volume Change in PPy(DBS): An Atomic Force Microscopy Study. Adv. Mater. 1999, 11, 953-956.

(15) Berdichevsky, Y.; Lo, Y. H. Polymer Microvalve Based on Anisotropic Expansion of Polypyrrole. In Micro- and Nanosystems; LaVan, D. A.; Ayon, A. A.; Madou, M. J.; McNie, M. E.; Prasad, S. V., Eds.; Materials Research Society: Warrendale, 2004; Vol. 782, pp. 101-107.

(16) Svennersten, K.; Berggren, M.; Richter-Dahlfors, A.; Jager, E. W. H. Mechanical Stimulation of Epithelial Cells Using Polypyrrole Microactuators. Lab. Chip 2011, 11, 3287-3293.

(17) Shimoda, S.; Smela, E. The Effect of pH on Polymerization and Volume Change in PPy(DBS). Electrochimica Acta 1998, 44, 219-238.

(18) Pei, Q.; Inganas, O. Electrochemical Applications of the Bending Beam Method .2. Electroshrinking and Slow Relaxation in Polypyrrole. J. Phys. Chem. 1993, 97, 6034-6041.

(19) Bay, L.; Mogensen, N.; Skaarup, S.; Sommer-Larsen, P.; Jorgensen, M.; West, K. Polypyrrole Doped with Alkyl Benzenesulfonates. Macromolecules 2002, 35, 9345-9351.

(20) Vetter, K. . Electrochemical Kinetics : Theoretical and Experimental Aspects; Academic Press: New York, N.Y, 1967.

(21) Martinez, J. G.; Otero, T. F. Biomimetic Dual SensingActuators: Theoretical Description. Sensing Electrolyte Concentration and Driving Current. J. Phys. Chem. B 2012, 116, 9223-9230.

(22) Potje-Kamloth, K. Chemical Gas Sensors Based on Organic Semiconductor Polypyrrole. Crit. Rev. Anal. Chem. 2002, 32, 121-140.

(23) Wei, D.; Bailey, M. J. A.; Andrew, P.; Ryhaenen, T. Electrochemical Biosensors at the Nanoscale. Lab. Chip 2009, 9, 2123-2131.

(24) Rover, L.; Neto, G. D.; Kubota, L. T. Potentiometric transducers based in conducting polymers: Analytical applications. Quimica Nova 1997, 20, 519-527.

(25) Dhand, C.; Das, M.; Datta, M.; Malhotra, B. D. Recent Advances in Polyaniline Based Biosensors. Biosens. Bioelectron. 2011, 26, 2811-2821.

(26) Laslau, C.; Williams, D. E.; Wright, B. E.; Travas-Sejdic, J. Measuring the Ionic Flux of an Electrochemically Actuated Conducting Polymer Using Modified Scanning Ion Conductance Microscopy. J. Am. Chem. Soc. 2011, 133, 57485751.

(27) Bay, L.; Jacobsen, T.; Skaarup, S.; West, K. Mechanism of Actuation in Conducting Polymers: Osmotic Expansion. J. Phys. Chem. B Chem. 2001, 105, 8492-8497.

(28) Carlsson, D.; Jager, E.; Krogh, M.; Skoglund, M. Systems, Device and Object Comprising Electroactive Polymer
Material, Methods and Uses Relating to Operation and Provision Thereof. WO 2009/038501 A1, 2009.

(29) Jo, C.; Naguib, H. E.; Kwon, R. H. Fabrication, Modeling and Optimization of an Ionic Polymer Gel Actuator. Smart Mater. Struct. 2011, 20, 045006.

(30) Jafeen, M. J. M.; Careem, M. A.; Skaarup, S. Speed and Strain of Polypyrrole Actuators: Dependence on Cation Hydration Number. Ionics 2010, 16, 1-6.

(31) Vidanapathirana, K. P.; Careem, M. A.; Skaarup, S.; West, K. Ion Movement in Polypyrrole/dodecylbenzenesulphonate Films in Aqueous and Non-Aqueous Electrolytes. Solid State Ion. 2002, 154, 331-335.

(32) Otero, T. F.; Alfaro, M.; Martinez, V.; Perez, M. A.; Martinez, J. G. Biomimetic Structural Electrochemistry from Conducting Polymers: Processes, Charges, and Energies. Coulovoltammetric Results from Films on Metals Revisited. Adv. Funct. Mater. 2013, 23, 3929-3940.

(33) Melling, D.; Wilson, S.; Jager, E. W. H. The Effect of Film Thickness on Polypyrrole Actuation Assessed Using Novel Non-Contact Strain Measurements. Smart Mater. Struct. 2013, 22, 104021.

(34) Chemistry of Nonaqueous Solutions: Current Progress.; Mamantov, G.; Popov, A. I., Eds.; Wiley-VCH: Michigan, 1994.

(35) Hara, S.; Zama, T.; Tanaka, N.; Takashima, W.; Kaneto, K. Artificial Fibular Muscles with 20\% Strain Based on Polypyrrole-Metal Coil Composites. Chem. Lett. 2005, 34, 784785.

(36) Hara, S.; Zama, T.; Takashima, W.; Kaneto, K. TFSI-Doped Polypyrrole Actuator with 26\% Strain. J. Mater. Chem. 2004, 14, 1516-1517.

(37) Otero, T. F.; Martinez, J. G. Artificial Muscles: A Tool To Quantify Exchanged Solvent during Biomimetic Reactions. Chem. Mater. 2012, 24, 4093-4099.

(38) Machu, W. Handbook of Electropainting Technology; Electrochemical Publications Limited: Glasgow, 1978.

(39) Otero, T. F.; Boyano, I. Nucleation and Nonstoichiometry in Electrochromic Conducting Polymers. Chemphyschem 2003, 4, 868-872.

(40) Otero, T. F.; Martinez, J. G. Structural and Biomimetic Chemical Kinetics: Kinetic Magnitudes Include Structural Information. Adv. Funct. Mater. 2013, 23, 404-416. 


\section{TABLES}

Table 1: Potentials where both anodic and cathodic current maxima are obtained in different electrolyte concentration once attained a stationary response (third cycle). Difference between anodic and cathodic potentials.

\begin{tabular}{|c|c|c|c|}
\hline $\begin{array}{c}\text { Concentration / } \\
\mathrm{M}\end{array}$ & $\begin{array}{c}\text { Anodic maximum / V } \\
\text { vs Ag/AgCl }\end{array}$ & $\begin{array}{c}\text { Cathodic maximum / V } \\
\text { vs Ag/AgCl }\end{array}$ & $\begin{array}{c}\text { Potential difference between anodic and } \\
\text { cathodic maxima / V }\end{array}$ \\
\hline 0.0375 & -0.305 & -0.634 & 0.329 \\
\hline 0.075 & -0.326 & -0.655 & 0.329 \\
\hline 0.15 & -0.361 & -0.574 & 0.149 \\
\hline 0.3 & -0.373 & -0.522 & 0.101 \\
\hline 0.6 & -0.370 & -0.471 & 0.097 \\
\hline 1 & -0.368 & -0.465 & 0.089 \\
\hline 2 & -0.359 & -0.448 & 0.159 \\
\hline 3 & -0.353 & -0.512 & \\
\hline
\end{tabular}

Table 2: Redox charge and maximum movement obtained during cyclic voltammetry shown in Fig. 4. Slope of the expansion versus the consumed charge during reduction from fig. $3 \mathrm{e}$

\begin{tabular}{|c|c|c|c|}
\hline $\begin{array}{c}\text { Concentration / } \\
\mathrm{M}\end{array}$ & $\begin{array}{c}\text { Redox charge during cyclic } \\
\text { voltammetry / } \mathrm{mC}\end{array}$ & $\begin{array}{c}\text { Total expansion } \\
/ \mu \mathrm{m}\end{array}$ & $\begin{array}{c}\text { Expansion per consumed charge (re- } \\
\text { duction) } / \mu \mathrm{mC}^{-1}\end{array}$ \\
\hline 0.0375 & 72.6 & 0.51 & -0.31 \\
\hline 0.075 & 79.0 & 0.64 & -0.33 \\
\hline 0.15 & 80.0 & 0.74 & -0.49 \\
\hline 0.3 & 79.9 & 0.78 & -0.41 \\
\hline 0.6 & 76.1 & 0.69 & -0.25 \\
\hline 1 & 73.8 & 0.46 & -0.19 \\
\hline 2 & 65.7 & 0.29 & -0.12 \\
\hline 3 & 57.8 & 0.18 & \\
\hline
\end{tabular}

\section{CAPTION FOR FIGURES}

Figure 1: A schematic picture describing the calculation of the metrics used: thickness (d) expansion $(\Delta \mathrm{d})$ and strain $(\varepsilon)$. $\mathrm{D}_{\mathrm{PPy}}$ : diameter of PPy(DBS) is arbitrary state; $\mathrm{D}_{\text {met }}$ : diameter of the Au or Pt wire; $\mathrm{D}_{\mathrm{ox}}$ : diameter of PPy in the oxidized (con- 
tracted) state; $\mathrm{D}_{\text {red }}$ : diameter of PPy in the reduced (expanded) state; $\mathrm{D}_{0}$ : initial diameter of the 'as-polymerized' PPy film on the metal wire.

Figure 2: (a) Voltammograms obtained from an uncoated gold wire (black line) and an uncoated platinum wire (red line) in $0.0375 \mathrm{M} \mathrm{NaCl}$ aqueous solution at $20 \mathrm{mV} \mathrm{s}^{-1}$. (b) Coulovoltammograms (accumulated consumed charge) obtained by integration of the voltammograms shown in (a) in the studied limits, $-1.25 \mathrm{~V}$ and $0.5 \mathrm{~V}$ versus $\mathrm{Ag} / \mathrm{AgCl}$.

Figure 3: (a) Voltammograms obtained from a PPy(DBS) coated gold wire before and after cycling the material with potential steps between $-1 \mathrm{~V}$ and $0 \mathrm{~V}$ versus $\mathrm{Ag} / \mathrm{AgCl}$, kept for $200 \mathrm{~s}$ every time in $0.0375 \mathrm{M} \mathrm{NaCl}$ aqueous solution. (b) Voltammograms obtained from a PPy(DBS) coated platinum wire before and after cycling the material with potential steps between $-0.8 \mathrm{~V}$ and $0 \mathrm{~V}$ versus $\mathrm{Ag} / \mathrm{AgCl}$, kept for $200 \mathrm{~s}$ every time in $0.0375 \mathrm{M} \mathrm{NaCl}$ aqueous solution. (c) Coulovoltammograms obtained by integration of the voltammograms shown in (a). (d)Coulovoltammograms obtained by integration of the voltammograms shown in (b). (e) Diameter change (in \%) suffered by a PPy(DBS) coated gold wire during voltammograms shown in (a). (f) Diametrical strain of the PPy layer (in \%) suffered by a PPy(DBS) coated platinum wire during voltammograms shown in (b). All the experiments in figure were carried out at room temperature.

Figure 4: (a) Stationary voltammetric responses obtained from a PPy(DBS) coated Pt wire in $\mathrm{NaCl}$ aqueous solutions (different concentrations, indicated in the figure) at $20 \mathrm{mV} \mathrm{s}^{-1}$. (b) Coulovoltammograms obtained by integration of the voltammograms shown in (a). (c) Expansion of the PPy(DBS) layer coated on Pt wire during cyclic voltammetry in (a). (d) Expansion from (c) versus consumed charge from (b).

Figure 5: Stationary anodic (a) and cathodic (b) chronoamperometric responses from a PPy(DBS) coated Pt wire in different concentrations (indicated in the figure) of $\mathrm{NaCl}$ aqueous solutions after submitted to 8 consecutive square potential steps from $-0.8 \mathrm{~V}$ versus $\mathrm{Ag} / \mathrm{AgCl}$, kept for $150 \mathrm{~s}$, to $0 \mathrm{~V}$, also kept for $150 \mathrm{~s}$. The insets show the initial three seconds. PPy expansion in the studied electrolytes during the oxidations (c) shown in (a), or during the reductions (d) shown in (b). Expansion rate vs time during oxidation (e) or reduction (f) responses. (g) Maximum expansion (open symbols), correlated consumed charge (solid symbols), and expansion rate (crosses) vs. concentrations during redox reactions (oxidation: black; reduction: red).

\section{FIGURES}

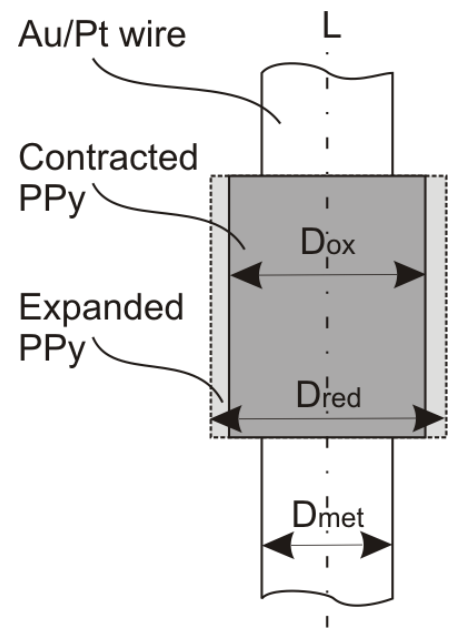

PPy thickness:

$d_{P P y}=\left(D_{\text {PPy }}-D_{\text {met }}\right) / 2$

\section{Expansion:}

$\Delta \mathrm{d}=\left(\mathrm{D}_{\mathrm{red}}-\mathrm{D}_{\mathrm{ox}}\right) / 2$

Strain (\%):

$\varepsilon=\left(D_{\text {red }}-D_{\text {ox }}\right) /\left(D_{0}-D_{\text {met }}\right) * 100 \%$

Figure 1 

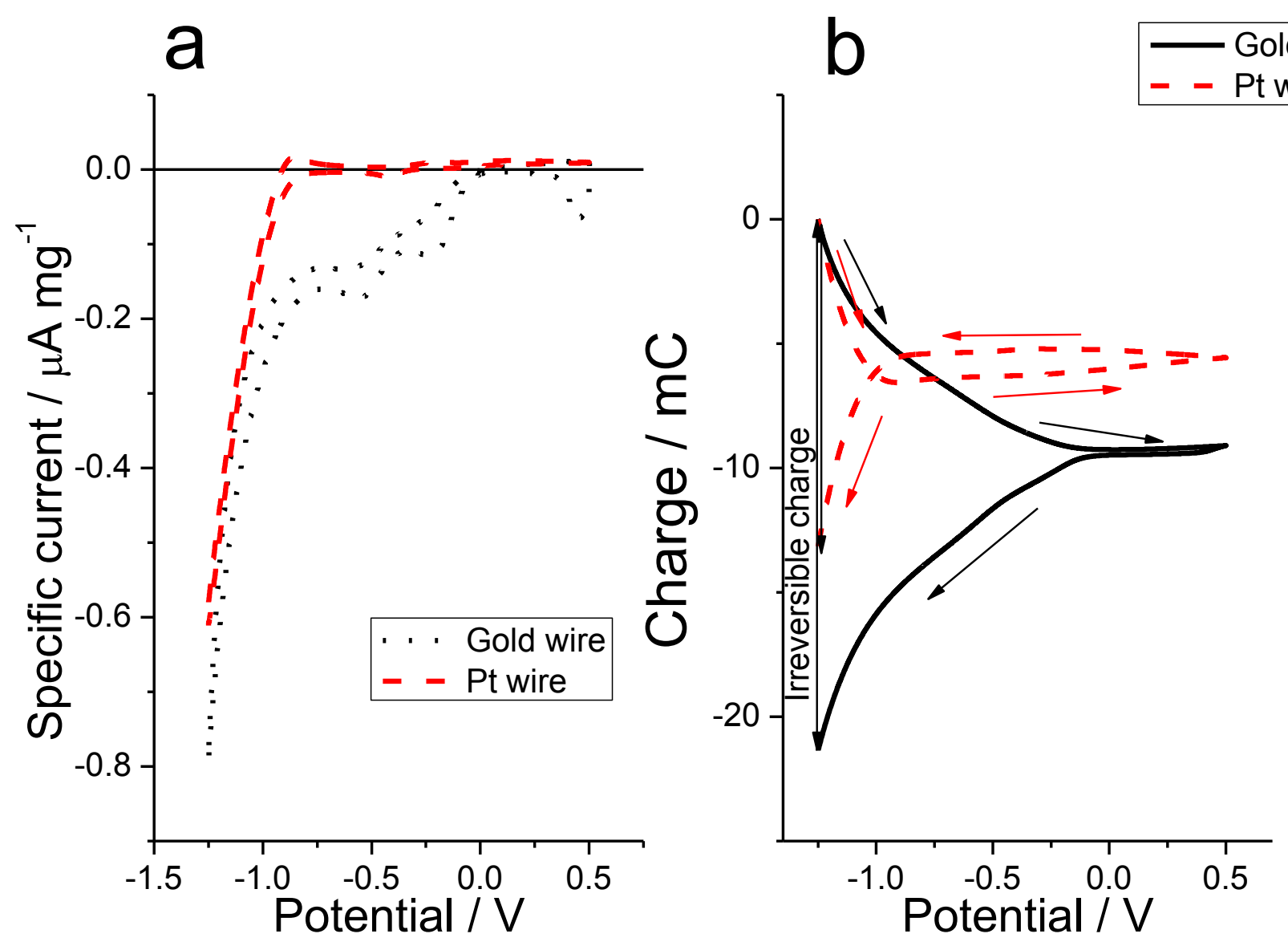

Figure 2 

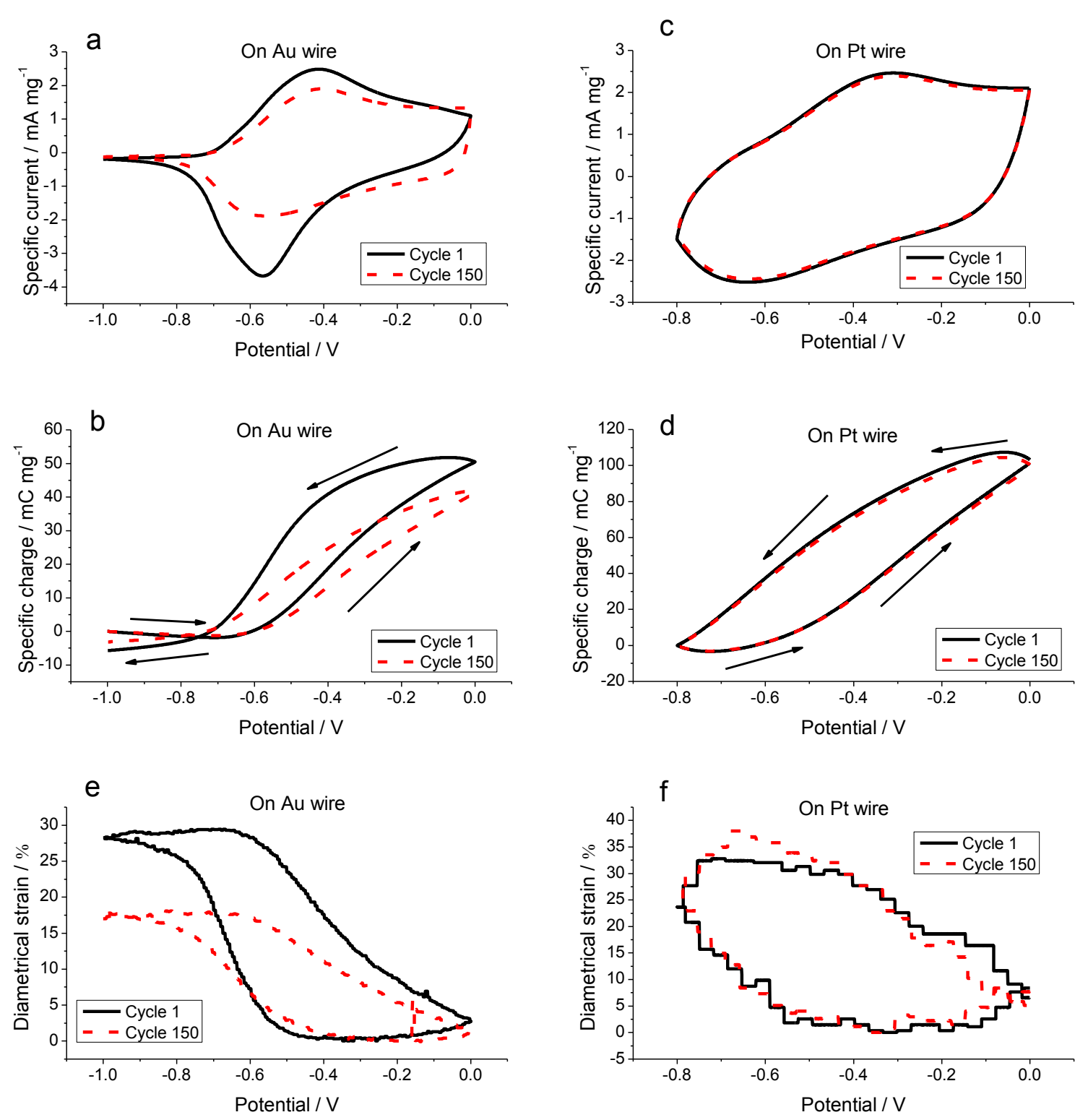

Figure 3 

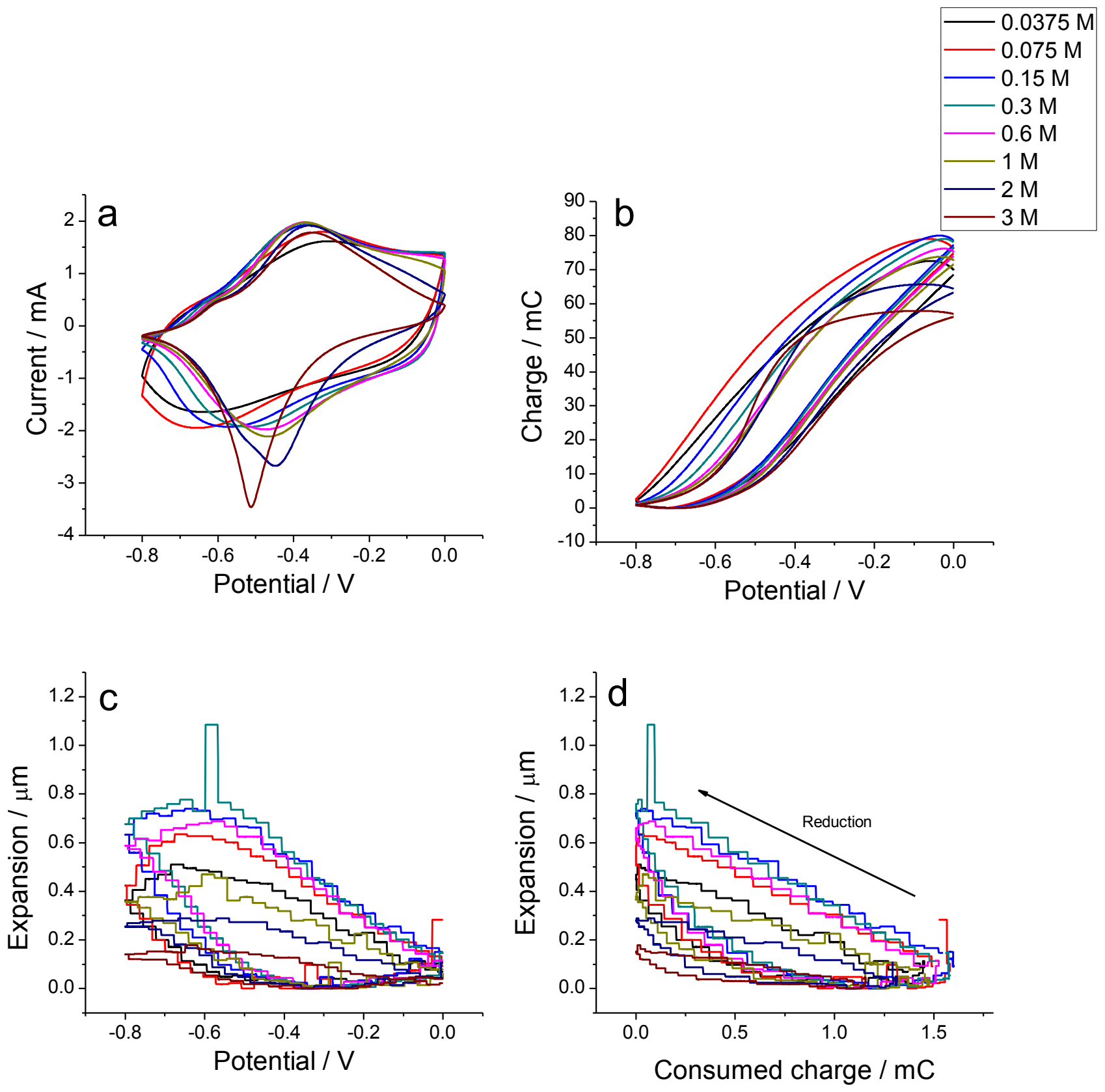

Figure 4 

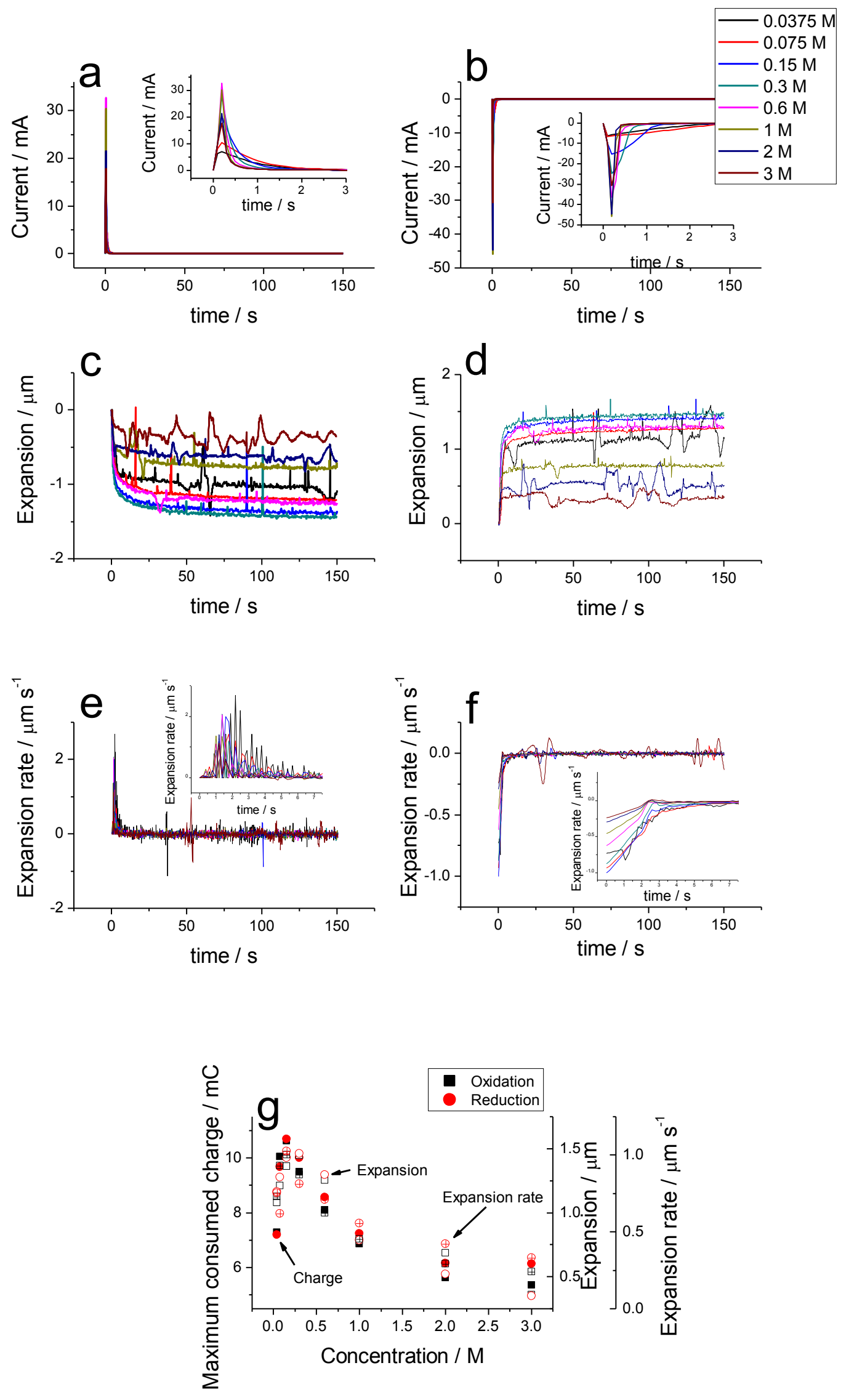

Figure 5 
TABLE OF CONTENTS

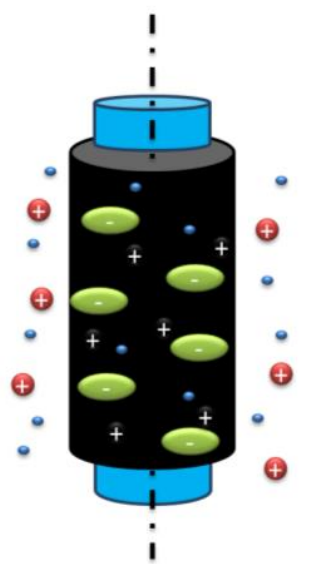

OXIDIZED PPy(DBS)

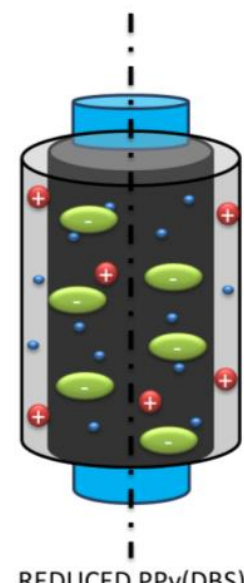

REDUCED PPy(DBS) 